\title{
On the adjacency algebras of near hexagons with an order
}

\author{
Bart De Bruyn
}

Received: date / Accepted: date

\begin{abstract}
Suppose $\mathcal{S}$ is a finite near hexagon of order $(s, t)$ having $v$ points. For every $i \in\{0,1,2,3\}$, let $A_{i}$ denote the adjacency matrix of the graph defined on the points by the distance $i$ relation. We perform a study of the real algebra generated by the $A_{i}$ 's, and take a closer look to the structure of these algebras for all known examples of $\mathcal{S}$. Among other things, we show that a certain number $d_{\mathcal{S}}$ (which is a function of $s, t$ and $v$ ) must be integral. This allows us to exclude certain near hexagons whose (non)existence was already open for about 15 years. In the special case $s=2$, we also show that the embedding rank of the near hexagon is at least the number $d_{\mathcal{S}}$, and that the near hexagon has non-full projective dimensions with vector dimension equal to $d_{\mathcal{S}}$.
\end{abstract}

Keywords: near hexagon, adjacency algebra, projective embedding MSC2010: 05E30, 05B25, 51A45, 51E12

\section{Introduction}

A point-line geometry $\mathcal{S}=(\mathcal{P}, \mathcal{L}, \mathrm{I})$ with non-empty point set $\mathcal{P}$, line set $\mathcal{L}$ and incidence relation $\mathrm{I} \subseteq \mathcal{P} \times \mathcal{L}$ is called a near polygon if the following properties hold.

(NP1) Every two distinct points are incident with at most one line.

(NP2) For every point $x$ and every line $L$, there exists a unique point on $L$ that is nearest to $x$ with respect to the distance $\mathrm{d}(\cdot, \cdot)$ in the collinearity graph $\Gamma$.

B. De Bruyn

Ghent University

Department of Mathematics

Krijgslaan 281 (S22)

9000 Gent (Belgium)

E-mail: Bart.DeBruyn@Ugent.be 
(NP3) The diameter of $\Gamma$ is finite.

If $d$ is the diameter of $\Gamma$, then the near polygon is called a near $2 d$-gon. In particular, the near polygon is a near quadrangle, hexagon or octagon depending on whether $d$ is equal to 2,3 or 4 . A near quadrangle in which every point is incident with at least two lines is also called a generalized quadrangle.

If $x$ is a point of a near polygon, then $\Gamma_{i}(x)$ with $i \in \mathbb{N}$ denotes the set of points at distance $i$ from $x$. A near polygon is said to have order $(s, t)$ if every line is incident with precisely $s+1$ points and if every point is incident with exactly $t+1$ lines. A near polygon is called dense if every line is incident with at least three points and if every two points at distance 2 have at least two common neighbours.

A set $Q$ of points of a near polygon $\mathcal{S}$ is called a quad if the following three properties are satisfied: (1) every line of $\mathcal{S}$ that contains two points of $Q$ has all its points in $Q ;(2)$ every point on a shortest path between two points of $Q$ is also contained in $Q ;(3)$ the point-line geometry defined on the point set $Q$ by those lines of $\mathcal{S}$ that have all their points in $Q$ is a generalized quadrangle. By Proposition 2.5 of [17, we know that every two points $x$ and $y$ of a dense near polygon at distance 2 are contained in a unique quad. We denote this quad by $\langle x, y\rangle$.

Suppose $\mathcal{S}=(\mathcal{P}, \mathcal{L}, \mathrm{I})$ is a finite near hexagon of order $(s, t)$ having $v=|\mathcal{P}|$ points. With respect to the point set $\mathcal{P}$, real $v \times v$ matrices $A_{i}, i \in\{0,1,2,3\}$, can be defined in the following way:

$$
\left(A_{i}\right)_{x y}= \begin{cases}1 & \text { if the points } x \text { and } y \text { are at distance } i, \\ 0 & \text { otherwise. }\end{cases}
$$

Then $A_{0}$ is equal to the $v \times v$ identity matrix $I$ and $A:=A_{1}$ is the collinearity matrix of $\mathcal{S}$.

Let $\mathbb{R}^{v \times v}$ denote the algebra of the real $v \times v$ matrices. The (commutative) subalgebra $\mathcal{A}$ of $\mathbb{R}^{v \times v}$ generated by $A$ will be called the adjacency algebra of $\mathcal{S}$. The subalgebra $\overline{\mathcal{A}}$ of $\mathbb{R}^{v \times v}$ generated by the matrices $A_{0}, A_{1}, A_{2}, A_{3}$ will be called the enlarged adjacency algebra of $\mathcal{S}$. In this paper, we prove the following results about these algebras.

Theorem 1 Let $A$ be the collinearity matrix of a finite near hexagon $\mathcal{S}$ of order $(s, t)$ having $v$ points. Then the following hold.

(1) The smallest eigenvalue of $A$ is equal to $-(t+1)$.

(2) The number

$$
d_{\mathcal{S}}:=\frac{s^{5} v}{(s+1)^{2}(s-1)\left(s^{2}+1\right)+s t(s-1)(s+1)^{2}+v}
$$

is integral.

(3) The multiplicity of the eigenvalue $-(t+1)$ of $A$ is at least $d_{\mathcal{S}}$.

Theorem 2 Suppose $\mathcal{S}$ is a finite near hexagon of order $(s, t)$ and denote by $\mathcal{A}$ and $\overline{\mathcal{A}}$ the adjacency and enlarged adjacency algebras of $\mathcal{S}$. Then the following hold: 
(1) $\overline{\mathcal{A}}$ is commutative,

(2) $\operatorname{dim}(\overline{\mathcal{A}})-\operatorname{dim}(\mathcal{A}) \in\{0,1\}$,

(3) $\overline{\mathcal{A}}=\mathcal{A}$ if and only if the multiplicity of the eigenvalue $-(t+1)$ of the adjacency matrix $A$ is equal to the number $d_{\mathcal{S}}$ defined in Theorem 1.

Theorems 1 and 2 will be proved in Sections 2 and 3 In Section 4 , we take a look at the (enlarged) adjacency algebras of all known finite near hexagons with an order. For each of these known examples, it turns out that both algebras coincide, or equivalently that the multiplicity of the eigenvalue $-(t+1)$ is equal to the number $d_{\mathcal{S}}$. We have however not succeeded in proving that this fact is valid for general finite near hexagons with an order. Another question which naturally arises is whether the above algebras are also closed under entry-wise product or Hadamard product of matrices (as it is the case for near hexagons with a distance-regular collinearity graph). It turns out that among the known examples, there is a unique near hexagon (namely $\mathbb{G}_{3}$ ) for which this is not the case.

We have initiated the study of the adjacency algebras of general near hexagons with an order in the hope that this will be a fruitful tool for the study of such near hexagons. Theorem 1(2) indeed has some consequences to the classification problem of the finite dense near polygons with four points per line. While those of diameter 2 (the generalized quadrangles with four points per line) have been classified by Dixmier and Zara 11 (see also Section 6.2 of [15), incomplete classifications have been obtained for the near hexagons and octagons, see [6,7].

There are up to isomorphism ten known examples of finite dense near hexagons with four points per line. These are described in [6] where it was also shown that every other (unknown) finite dense near hexagon $\mathcal{S}$ with four points per line can only have quads that are $(3 \times 3)$-grids or isomorphic to the generalized quadrangle $Q(4,3)$ (as defined in 15, for instance). There exist moreover constants $t, a, b$ such that $\mathcal{S}$ has order $(3, t)$ and every point is contained in precisely $a$ grid-quads and $b Q(4,3)$-quads. From Theorem 4.17 of [6], we know that $(v, t, a, b)$ is among the following four possibilities:

$(5848,19,160,5), \quad(6736,21,171,10), \quad(8320,27,120,43), \quad(20608,34,595,0)$.

In Proposition 4.7 of [10], it was shown that the last case cannot occur. By (2) of Theorem 1 we now also know that the first two cases cannot occur. We can conclude the following.

Corollary 1 Suppose $\mathcal{S}$ is a finite dense near hexagon with four points per line that is not among the ten known examples described in [6]. Then $\mathcal{S}$ has order $(3,27)$ and contains 8320 points. Moreover, every point of $\mathcal{S}$ is contained in precisely 120 grid-quads and 43 Q(4,3)-quads.

A near hexagon of order $(3,27)$ having the properties stated in Corollary 1 will be called an exceptional near hexagon. In [7, we obtained an incomplete classification for the finite dense near octagons with four points per line. 
We showed there that each such near octagon either belongs to a list of 28 known examples, contains an (unknown) dense near hexagon with parameters $(v, s, t, a, b)=(20608,3,34,595,0)$ as subgeometry, or is a direct product of a line of size 4 with an (unknown) dense near hexagon with four points per line. Combining this with Proposition 4.7 of [10] and Corollary 1 of the present paper, we obtain:

Corollary 2 Suppose $\mathcal{S}$ is a finite dense near octagon with four points per line that is not among the 28 known examples mentioned in Proposition 1.1 of [7]. Then $\mathcal{S}$ is the direct product of an exceptional near hexagon $\mathbb{E}$ of order $(3,27)$ and a line of size 4 .

Note that such a direct product is obtained by stacking four isomorphic copies of $\mathbb{E}$ and joining the corresponding points by lines of size 4 .

Theorem 1(3) has some consequences to full projective embeddings of near hexagons of order $(2, t)$. A projective embedding of a point-line geometry $\mathcal{S}=$ $(\mathcal{P}, \mathcal{L}, \mathrm{I})$ is defined as an injective map $\epsilon$ from $\mathcal{P}$ to the point set of a projective space $\Sigma$ mapping every line of $\mathcal{S}$ into some line of $\Sigma$ such that $\langle\epsilon(\mathcal{P})\rangle_{\Sigma}=\Sigma$. We denote such an embedding by $\epsilon: \mathcal{S} \rightarrow \Sigma$, and $\overline{\operatorname{Im}(\epsilon)}$ denotes the set of all points of $\Sigma$ that are contained in $\operatorname{Im}(\epsilon)=\epsilon(\mathcal{P})$ or on a line joining two points of $\operatorname{Im}(\epsilon)$. If every line of $\mathcal{S}$ is mapped to a complete line of $\Sigma$, then the embedding is called full, otherwise it is called non-full.

Two projective embeddings $\epsilon_{1}: \mathcal{S} \rightarrow \Sigma_{1}$ and $\epsilon_{2}: \mathcal{S} \rightarrow \Sigma_{2}$ are called isomorphic if there exists an isomorphism $\phi: \Sigma_{1} \rightarrow \Sigma_{2}$ such that $\epsilon_{2}=\phi \circ \epsilon_{1}$.

Suppose $\epsilon: \mathcal{S} \rightarrow \Sigma$ is a full projective embedding of $\mathcal{S}$ and $\alpha$ is a subspace of $\Sigma$ disjoint from $\overline{\operatorname{Im}(\epsilon)}$. Then a new full projective embedding $\epsilon / \alpha: \mathcal{S} \rightarrow \Sigma / \alpha$ can be defined which maps each point $x$ of $\mathcal{S}$ to the point $\langle\alpha, \epsilon(x)\rangle$ of the quotient projective space $\Sigma / \alpha$. We call $\epsilon / \alpha$ a quotient of $\epsilon$. A full projective embedding $\widetilde{\epsilon}: \mathcal{S} \rightarrow \widetilde{\Sigma}$ is called absolutely universal if every full projective embedding of $\mathcal{S}$ is isomorphic to a quotient of $\tilde{\epsilon}$.

Suppose $\epsilon: \mathcal{S} \rightarrow \mathrm{PG}(V)$ is a full projective embedding of a point-line geometry $\mathcal{S}$ with three points per line. Then by Ronan [16], we know that $\mathcal{S}$ has an absolutely universal embedding $\widetilde{\epsilon}: \mathcal{S} \rightarrow \mathrm{PG}(\widetilde{V})$, which is moreover unique up to isomorphism. The vector $\operatorname{dimension} \operatorname{dim}(\widetilde{V})$ of $\widetilde{\epsilon}$ is called the embedding rank of $\mathcal{S}$. There are 15 known examples of finite near hexagons $\mathcal{S}$ with three points per line that have an order, and for each of them, it turns out that the embedding rank is equal to the number $d_{\mathcal{S}}$. In fact, we are able to prove the following, see Section 5 .

Theorem 3 Suppose $\mathcal{S}$ is a finite near hexagon of order $(2, t)$. Then $\mathcal{S}$ has full projective embeddings, and the embedding rank $r$ of $\mathcal{S}$ is at least $d_{\mathcal{S}}$. If $r=d_{\mathcal{S}}$, then $d_{\mathcal{S}}$ is also equal to the multiplicity of the eigenvalue $-(t+1)$ of the collinearity matrix $A$.

Suppose $\mathcal{S}$ is a finite near hexagon of order $(2, t)$. Although we were not able to show that the embedding rank of $\mathcal{S}$ is equal to $d_{\mathcal{S}}$ or even just that $\mathcal{S}$ has 
full projective embeddings with vector dimension $d_{\mathcal{S}}$, it is possible to show that $\mathcal{S}$ has non-full projective embeddings with vector dimension equal to $d_{\mathcal{S}}$.

Theorem 4 Suppose $\mathcal{S}$ is a finite near hexagon of order $(2, t)$. Then $\mathcal{S}$ has a non-full projective embedding in $\mathrm{PG}\left(d_{\mathcal{S}}-1, \mathbb{R}\right)$.

\section{On finite near polygons with an order}

In this section, $\mathcal{S}=(\mathcal{P}, \mathcal{L}, \mathrm{I})$ denotes a finite near $2 d$-gon, $d \geq 1$, of order $(s, t)$. With respect to the sets $\mathcal{P}$ and $\mathcal{L}$, we can define matrices $A_{0}, A_{1}, \ldots, A_{d}$ (similarly as in Section 1), as well as the following matrix $M$ :

$$
M_{x, L}= \begin{cases}1 & \text { if the point } x \text { is incident with the line } L, \\ 0 & \text { otherwise. }\end{cases}
$$

Then $M$ is the incidence matrix of $\mathcal{S}$, and $A:=A_{1}$ is the collinearity matrix of $\mathcal{S}$. The matrix $A_{0}$ equals $I$, the $v \times v$ identity matrix. We also define

$$
N:=\sum_{i=0}^{d}\left(-\frac{1}{s}\right)^{i} A_{i} .
$$

Proposition 1 The smallest eigenvalue of $A$ is equal to $-(t+1)$. Every column of $N$ is an eigenvector of $A$ with eigenvalue $-(t+1)$.

Proof. We have $M M^{T}=(t+1) I+A$. If $m$ is the smallest eigenvalue of $A$, then $(t+1)+m$ is the smallest eigenvalue of $M M^{T}$. As $M M^{T}$ is positive semidefinite, we have $t+1+m \geq 0$, i.e. $m \geq-(t+1)$. We now show that $A N=$ $-(t+1) N$, i.e. every column of $N$ is an eigenvector of $A$ with corresponding eigenvalue $-(t+1)$, implying that $-(t+1)$ is the smallest eigenvalue of $A$.

Let $x$ and $y$ be two arbitrary points of $\mathcal{S}$, and put $j:=\mathrm{d}(x, y)$. Let $t_{j}+1$ denote the number of lines through $x$ containing a point at distance $j-1$ from $y$. Then among the $s(t+1)$ neighbors of $x, t_{j}+1$ lie at distance $j-1$ from $y,(s-1)\left(t_{j}+1\right)$ lie at distance $j$ from $y$ and $s\left(t-t_{j}\right)$ lie at distance $j+1$ from $y$. So, $(A N)_{x y}=\sum_{z \in \mathcal{P}} A_{x z} N_{z y}=\left(-\frac{1}{s}\right)^{j-1} \cdot\left(t_{j}+1\right)+\left(-\frac{1}{s}\right)^{j} \cdot(s-1)\left(t_{j}+\right.$ $1)+\left(-\frac{1}{s}\right)^{j+1} \cdot s\left(t-t_{j}\right)=-\left(-\frac{1}{s}\right)^{j} \cdot(t+1) \stackrel{s}{=}-(t+1) N_{x y}$. As this holds for all points $x$ and $y$ of $\mathcal{S}$, we indeed have $A N=-(t+1) N$, as we wished to prove.

\section{Proofs of Theorems 1 and 2}

In this section, we suppose that $\mathcal{S}=(\mathcal{P}, \mathcal{L}, \mathrm{I})$ is a finite near hexagon of order $(s, t)$ having $v$ points. The aim of this section is to prove Theorems 1 and 2 . Note that the first claim of Theorem 1 has already been proved in Proposition 1. 
Consider again the matrices $A_{0}, A_{1}, A_{2}, A_{3}$ as defined in Section 1 , and let $N$ be as defined in equation (1) (with $d=3$ ). In Proposition 4.1 of [8], we showed that

$$
N^{2}=\alpha N
$$

where

$$
\alpha:=\frac{(s+1)^{2}(s-1)\left(s^{2}+1\right)+s t(s-1)(s+1)^{2}+v}{s^{5}}>0 .
$$

Equation (2) implies that every eigenvalue of $N$ is either 0 or $\alpha$. If $m$ is the multiplicity of the eigenvalue $\alpha$, then $m \alpha=\operatorname{Tr}(N)=v$, and hence

$$
m=\frac{s^{5} v}{(s+1)^{2}(s-1)\left(s^{2}+1\right)+s t(s-1)(s+1)^{2}+v}=d_{\mathcal{S}} \in \mathbb{N} .
$$

Note that $m=d_{\mathcal{S}}$ is also equal to the rank of the matrix $N$. Since every column of $N$ is an eigenvector of $A$ with corresponding eigenvalue $-(t+1)$, we see that the multiplicity of the eigenvalue $-(t+1)$ is at least the number $m$. Thus we have also proved the two other claims of Theorem 1 .

By Proposition 1, we know that $A N=-(t+1) N$. Since $A$ and $N$ are symmetric matrices, we then have

$$
N A=N^{T} A^{T}=(A N)^{T}=-(t+1) N^{T}=-(t+1) N=A N .
$$

Denote by $J$ the real $v \times v$ matrix with all entries equal to 1 . Since the collinearity graph $\Gamma$ of $\mathcal{S}$ is regular and connected, we know from Theorem 1 of [12], that there exists a polynomial $p^{*}(x) \in \mathbb{Q}[x]$ such that $J=p^{*}(A)$. We then have

$$
A_{3}=p^{*}(A)-I-A-A_{2} .
$$

From $N=I-\frac{A}{s}+\frac{A_{2}}{s^{2}}-\frac{A_{3}}{s^{3}}$ and $I+A+A_{2}+A_{3}=J=p^{*}(A)$, it follows that

$$
(s+1) A_{2}=p^{*}(A)+\left(s^{2}-1\right) A-\left(s^{3}+1\right) I+s^{3} N .
$$

Equations (4) and (5) imply that the enlarged adjacency algebra $\overline{\mathcal{A}}$ of $\mathcal{S}$ coincides with the subalgebra of $\mathbb{R}^{v \times v}$ generated by $A$ and $N$. By equations $(2)$ and (3), we then know that the algebra $\overline{\mathcal{A}}$ is commutative and that $\overline{\mathcal{A}}=\langle\overrightarrow{\mathcal{A}}, N\rangle$. The latter implies that $\operatorname{dim}(\overline{\mathcal{A}})-\operatorname{dim}(\mathcal{A}) \in\{0,1\}$. We have thus proved Claims (1) and (2) of Theorem 2 Since the matrices $A_{0}, A_{1}, A_{2}, A_{3} \in \overline{\mathcal{A}}$ commute, we know that the following holds.

Corollary $3 \mathbb{R}^{v}$ has a basis consisting of common eigenvectors of the matrices $A_{0}, A_{1}, A_{2}$ and $A_{3}$.

We now prove the third claim of Theorem 2 Since $\overline{\mathcal{A}}=\langle\mathcal{A}, N\rangle$, we see that $\overline{\mathcal{A}}=\mathcal{A}$ if and only if $N=p_{N}(A)$ for some polynomial $p_{N}(x) \in \mathbb{R}[X]$.

Suppose $N=p_{N}(A)$ for some polynomial $p_{N}(x) \in \mathbb{R}[x]$. Since $(A+(t+$ 1) $I) N=0$, the polynomial $(x+t+1) p_{N}(x)$ annihilates the matrix $A$ and so should be divisible by the minimal polynomial $m(x)$ of $A$. Every eigenvalue of $A$ distinct from $-(t+1)$ is thus a root of $p_{N}(x)$. If also $-(t+1)$ is a root of 
$p_{N}(x)$, then $N=p_{N}(A)$ would be the zero matrix, an obvious contradiction. So, all eigenvalues of $N=p_{N}(A)$ are zero, except for the eigenvalue $p_{N}(-t-1)$ whose multiplicity is equal to the multiplicity of the eigenvalue $-t-1$ of $A$. The multiplicity must be equal to the rank $d_{\mathcal{S}}$ of $N$.

Conversely, suppose that the multiplicity of $-t-1$ as eigenvalue of $A$ is equal to the number $d_{\mathcal{S}}$. By Corollary 3, there exists an invertible real $v \times v$ matrix such that $\widetilde{A}:=Q^{-1} A Q$ and $\widetilde{N}:=Q^{-1} N Q$ are diagonal matrices. Now, $(\widetilde{A}+(t+1) I) \widetilde{N}=0$, where $\widetilde{A}+(t+1) I$ is a diagonal matrix of rank $v-d_{\mathcal{S}}$ and $\tilde{N}$ is a diagonal matrix of rank $d_{\mathcal{S}}$ whose diagonal entries are 0 or $\alpha$ (as 0 and $\alpha$ are the only eigenvalues of $N$ ). We conclude the following:

The positions where $-t-1$ occurs in the matrix $\widetilde{A}$ are precisely the positions where $\alpha$ occurs in $\widetilde{N}$.

Now, let $p_{N}(x) \in \mathbb{R}[x]$ be a polynomial that maps $-(t+1)$ to $\alpha$ and every other eigenvalue of $A$ to 0 . Then, $\widetilde{N}=p_{N}(\widetilde{A})$ and hence also $N=p_{N}(A)$.

\section{Examples}

In this section, we take a look at the (enlarged) adjacency algebras of all known finite near hexagons with an order. In particular, we determine their dimensions and verify whether they are closed under Hadamard multiplication of matrices.

\subsection{Regular near hexagons}

A finite near hexagon $\mathcal{S}$ is called regular if there exist constants $s, t, t_{2}$ such that $\mathcal{S}$ has order $(s, t)$ and every two points at distance 2 from each other have precisely $t_{2}+1$ common neighbours. If this is the case, then the number $v$

of points of $\mathcal{S}$ is equal to $(s+1)\left(1+s t+\frac{s^{2} t\left(t-t_{2}\right)}{t_{2}+1}\right)$. A finite near hexagon is regular precisely when its collinearity graph is distance-regular [3]. For regular near hexagons, we know from [3, 4 that $\overline{\mathcal{A}}=\mathcal{A}$ and that the multiplicity of the eigenvalue $-(t+1)$ is equal to $d_{\mathcal{S}}=s^{3} \frac{\left(t_{2}+1\right)+s\left(t_{2}+1\right) t+s^{2} t\left(t-t_{2}\right)}{s^{2}\left(t_{2}+1\right)+s t\left(t_{2}+1\right)+t\left(t-t_{2}\right)}$.

\subsection{The near hexagon $\mathbb{H}_{3}$}

The near hexagon $\mathbb{H}_{3}$ belongs to an infinite family of near polygons described in [2]. The points of $\mathbb{H}_{3}$ are the partitions of $\{1,2, \ldots, 8\}$ into four pairs, the lines of $\mathbb{H}_{3}$ are the partitions of $\{1,2, \ldots, 8\}$ into one subset of size 4 and two pairs, with a point $x$ being incident with a line $L$ if $x$ (regarded as a partition) is a refinement of $L$. The geometry $\mathbb{H}_{3}$ is a near hexagon of order $(2,5)$ and has $v=105$ points, implying that $d_{\mathbb{H}_{3}}=14$. 
The group $S_{8}$ has a natural action on the point set $\mathcal{P}$ of $\mathbb{H}_{3}$, and this action gives rise to the full automorphism group of $\mathbb{H}_{3}$. The action of $S_{8}$ on $\mathcal{P}$ is transitive and there are five orbitals corresponding to the following relations:

$$
\begin{aligned}
R_{0} & :=\{(x, x) \mid x \in \mathcal{P}\} ; \\
R_{1} & :=\{(x, y) \in \mathcal{P} \times \mathcal{P} \mid \mathrm{d}(x, y)=1\} ; \\
R_{2 a} & :=\left\{(x, y) \in \mathcal{P} \times \mathcal{P} \mid \mathrm{d}(x, y)=2 \text { and }\left|\Gamma_{1}(x) \cap \Gamma_{1}(y)\right|=2\right\} ; \\
R_{2 b} & :=\left\{(x, y) \in \mathcal{P} \times \mathcal{P} \mid \mathrm{d}(x, y)=2 \text { and }\left|\Gamma_{1}(x) \cap \Gamma_{1}(y)\right|=3\right\} ; \\
R_{3} & :=\{(x, y) \in \mathcal{P} \times \mathcal{P} \mid \mathrm{d}(x, y)=3\} .
\end{aligned}
$$

The relation $R_{*}$ defines a graph on the vertex set $\mathcal{P}$ of $\mathbb{H}_{3}$ whose adjacency matrix will be denoted by $A_{*}$. Then $A_{0}=I=I_{v}$. Putting $A=A_{1}$, a straightforward computation shows that $A^{2}=12 I+A+2 A_{2 a}+3 A_{2 b}, A A_{2 a}=$ $2 A+2 A_{2 a}+2 A_{3}, A A_{2 b}=8 A+3 A_{2 b}+4 A_{3}$ and $A A_{3}=8 A_{2 a}+6 A_{2 b}+6 A_{3}$. These equations can be summarized as

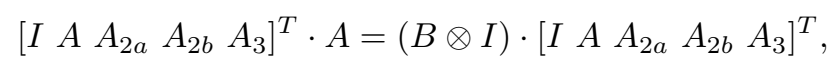

where $B \otimes I$ denotes the Kronecker product ([13, Section 4.2]) of

$$
B=\left[\begin{array}{ccccc}
0 & 1 & 0 & 0 & 0 \\
12 & 1 & 2 & 3 & 0 \\
0 & 2 & 2 & 0 & 2 \\
0 & 8 & 0 & 3 & 4 \\
0 & 0 & 8 & 6 & 6
\end{array}\right]
$$

and $I=I_{v}$. Let $\mathcal{A}$ be the subalgebra of $\mathbb{R}^{v \times v}$ generated by $A$, and let $\mathcal{B}$ be the subalgebra of $\mathbb{R}^{5 \times 5}$ generated by $B$. For every $M \in \mathcal{A}$, there exists a unique

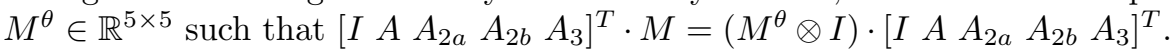
In fact, if $M=p(A)$ for a certain polynomial $p(x) \in \mathbb{R}[x]$, then we can take $M^{\theta}=p(B)$ by Lemma 4.2 .10 of 13 . The uniqueness of $M^{\theta} \in \mathbb{R}^{5 \times 5}$ follows from the fact that the matrices $I, A, A_{2 a}, A_{2 b}$ and $A_{3}$ are linearly independent in $\mathbb{R}^{v \times v}$. We conclude that $\theta$ defines an isomorphism between $\mathcal{A}$ and $\mathcal{B}$. The matrices $A$ and $B$ thus have the same eigenvalues, namely $\lambda_{1}=-6, \lambda_{2}=-1$, $\lambda_{3}=2, \lambda_{4}=5$ and $\lambda_{5}=12$.

If $j \in\{0,1,2,3,4\}$, then by (6), we can write $A^{j}=a_{j} I+b_{j} A_{1}+c_{j} A_{2 a}+$ $d_{j} A_{2 b}+e_{j} A_{3}$ for certain (necessarily unique) $a_{j}, b_{j}, c_{j}, d_{j}, e_{j} \in \mathbb{N}$. We then have $\operatorname{Tr}\left(A^{j}\right)=v a_{j}$. If $m_{i}$ with $i \in\{1,2,3,4,5\}$ is the multiplicity of $\lambda_{i}$ regarded as an eigenvalue of $A$, then for every $j \in\{0,1,2,3,4\}$, we have $\sum_{i=1}^{5} m_{i} \lambda_{i}^{j}=$ $\operatorname{Tr}\left(A^{j}\right)=v a_{j}$. These equations determine a system of linear equations which can be solved for the unknowns $m_{1}, \ldots, m_{5}$, as the matrix of the system is a nonsingular Vandermonde matrix. We find (e.g. with Maple) that $m_{1}=14$, $m_{2}=56, m_{3}=14, m_{4}=20$ and $m_{5}=1$. Note that $m_{1}=d_{\mathbb{H}_{3}}$ and thus that $\mathcal{A}=\overline{\mathcal{A}}$. As $A$ is a symmetric matrix having five distinct eigenvalues, we have $\operatorname{dim}(\mathcal{A})=5$. By $(6), \mathcal{A}$ is contained in and hence coincides with the 5dimensional subspace $\left\langle A_{0}, A_{1}, A_{2 a}, A_{2 b}, A_{3}\right\rangle$ of $\mathbb{R}^{v \times v}$. Since the latter is closed under Hadamard multiplication of matrices, the same holds for the adjacency algebra $\mathcal{A}$. 


\subsection{Other examples}

Every known finite non-regular near hexagon with an order $(s, t)$ is either a product near hexagon (as defined in 4]), a glued near hexagon (as defined in [5]) or is isomorphic to $\mathbb{H}_{3}, \mathbb{E}_{3}$ or $\mathbb{G}_{3}$. For each of these cases, it turns out that the multiplicity of the smallest eigenvalue $-(t+1)$ is equal to the number $d_{\mathcal{S}}$, and thus that $\overline{\mathcal{A}}=\mathcal{A}$. In each case, the verification of this fact can be done with similar computations as in Section 4.2 where we treated the near hexagon $\mathbb{H}_{3}$. We therefore only mention the outcome of the computations here. More information about the computations can be found in [9].

\section{The near hexagon $\mathbb{E}_{3}$}

The near hexagon $\mathbb{E}_{3}$ of order $(2,14)$ on 567 points was discovered by Aschbacher [1]. Other constructions can be found in [2] and [4. The eigenvalues of $A$ are $-(t+1)=-15,-3,3,9$ and 30 , with respective multiplicities $d_{\mathbb{E}_{3}}=21,315,140,90$ and 1 . As in the case of the near hexagon $\mathbb{H}_{3}$, the automorphism group is point-transitive and there are five orbitals, corresponding to relations $R_{0}, R_{1}, R_{2 a}, R_{2 b}$ and $R_{3}$. It is thus again the case that $\overline{\mathcal{A}}=\mathcal{A}=\left\langle A_{0}, A_{1}, A_{2 a}, A_{2 b}, A_{3}\right\rangle$ is closed under Hadamard multiplication.

The near hexagon $\mathbb{G}_{3}$

The near hexagon $\mathbb{G}_{3}$ of order $(2,11)$ on 405 points was constructed in [2]. The eigenvalues of $A$ are $-(t+1)=-12,-3,0,3,6,9$ and 24 , with respective multiplicities $d_{\mathbb{G}_{3}}=20,175,60,90,20,39$ and 1 . The algebra $\overline{\mathcal{A}}=\mathcal{A}$ is thus 7-dimensional. The automorphism group of $\mathbb{G}_{3}$ is point-transitive, and there are 10 orbitals, corresponding to relations $R_{0}, R_{1 a}, R_{1 b}, R_{2 a}, R_{2 b}, R_{2 c}, R_{2 d}$, $R_{3 a}, R_{3 b}$ and $R_{3 c}$. From the equation $\left[\begin{array}{lllll}I & A_{1 a} & A_{1 b} & \cdots & A_{3 c}\end{array}\right]^{T} \cdot A=(B \otimes I) \cdot$ $\left[\begin{array}{lllll}I & A_{1 a} & A_{1 b} & \cdots & A_{3 c}\end{array}\right]^{T}$ (being the equivalent of $\left[6\right.$ for the near hexagon $\mathbb{G}_{3}$ ), it can readily be derived (see [9]) that the smallest algebra generated by $A$ that is moreover closed under Hadamard multiplication coincides with the 10dimensional algebra generated by $A_{0}, A_{1 a}, A_{1 b}, A_{2 a}, A_{2 b}, A_{2 c}, A_{2 d}, A_{3 a}, A_{3 b}$ and $A_{3 c} . \mathcal{A}$ is itself thus not closed under Hadamard multiplication.

\section{Product near hexagons}

Suppose $\mathcal{S}$ is the direct product of a generalized quadrangle of order $\left(s, t_{2}\right)$ with a line of size $s+1$. We may assume that $t_{2} \geq 2$, otherwise $\mathcal{S}$ would be a Hamming near hexagon $([3])$ and hence regular. $\mathcal{S}$ has order $\left(s, t_{2}+1\right)$ and contains $(s+1)^{2}\left(s t_{2}+1\right)$ points. Let $\mathcal{L}$ denote the set of all lines of $\mathcal{S}$ not contained in any quad of order $\left(s, t_{2}\right)$, and consider the following relations on the point set $\mathcal{P}$ of $\mathcal{S}$ :

- $R_{0}=\{(x, x) \mid x \in \mathcal{P}\}$

- $R_{1 a}=\{(x, y) \in \mathcal{P} \times \mathcal{P} \mid \mathrm{d}(x, y)=1$ and $x y \in \mathcal{L}\}$;

- $R_{1 b}=\{(x, y) \in \mathcal{P} \times \mathcal{P} \mid \mathrm{d}(x, y)=1$ and $x y \notin \mathcal{L}\}$; 
- $R_{2 a}=\left\{(x, y) \in \mathcal{P} \times \mathcal{P} \mid \mathrm{d}(x, y)=2\right.$ and $\left.\left|\Gamma_{1}(x) \cap \Gamma_{1}(y)\right|=t_{2}+1\right\}$;

- $R_{2 b}=\left\{(x, y) \in \mathcal{P} \times \mathcal{P} \mid \mathrm{d}(x, y)=2\right.$ and $\left.\left|\Gamma_{1}(x) \cap \Gamma_{1}(y)\right|=2\right\}$;

- $R_{3}=\{(x, y) \in \mathcal{P} \times \mathcal{P} \mid \mathrm{d}(x, y)=3\}$.

The collinearity matrix $A$ has eigenvalues $-(t+1)=-t_{2}-2,-t_{2}-1+s$, $s-2,2 s-1, s t_{2}+s-1$ and $s t_{2}+2 s$, with respective multiplicities $d_{\mathcal{S}}=$ $\frac{s^{3}\left(s t_{2}+1\right)}{s+t_{2}}, \frac{\left(s t_{2}+1\right) s^{2}}{s+t_{2}}, \frac{(s+1)\left(t_{2}+1\right) s^{2} t_{2}}{s+t_{2}}, \frac{(s+1)\left(t_{2}+1\right) s t_{2}}{s+t_{2}}, s$ and 1. Moreover, $\overline{\mathcal{A}}=\mathcal{A}=$ $\left\langle A_{0}^{s+t_{2}}, A_{1 a}, A_{1 b}, A_{2 a}, A_{2 b}, A_{3}^{s+t_{2}}\right.$ is a 6 -dimensional algebra closed under Hadamard multiplication.

\section{Glued near hexagons}

Let $\mathcal{S}=(\mathcal{P}, \mathcal{L}, \mathrm{I})$ be a finite glued near hexagon. Then there exist two partitions $\mathcal{T}_{a}$ and $\mathcal{T}_{b}$ of $\mathcal{P}$ in isomorphic quads such that every quad of $\mathcal{T}_{a}$ intersects every quad of $\mathcal{T}_{b}$ in a line. We denote by $\mathcal{L}$ the set of all lines that arise in this way. We suppose that all quads of $\mathcal{T}_{a}$ have order $\left(s, t_{a}\right)$ and that all quads of $\mathcal{T}_{b}$ have order $\left(s, t_{b}\right)$. We may assume that $\mathcal{S}$ is not a product near hexagon which implies that $t_{a}, t_{b} \geq 2$. The near hexagon $\mathcal{S}$ has order $\left(s, t_{a}+t_{b}\right)$ and contains $(s+1)\left(s t_{a}+1\right)\left(s t_{b}+1\right)$ points. We define the following relations on the point set $\mathcal{P}$ of $\mathcal{S}$ :

- $\mathcal{R}_{0}=\{(x, x) \mid x \in \mathcal{P}\}$;

- $\mathcal{R}_{1 a}=\left\{(x, y) \in \mathcal{P} \times \mathcal{P} \mid \mathrm{d}(x, y)=1, x y \notin \mathcal{L}\right.$ and $x y$ is contained in a quad of $\left.\mathcal{T}_{a}\right\}$

- $\mathcal{R}_{1 b}=\left\{(x, y) \in \mathcal{P} \times \mathcal{P} \mid \mathrm{d}(x, y)=1, x y \notin \mathcal{L}\right.$ and $x y$ is contained in a quad of $\left.\mathcal{T}_{b}\right\} ;$

- $\mathcal{R}_{1 c}=\{(x, y) \in \mathcal{P} \times \mathcal{P} \mid \mathrm{d}(x, y)=1, x y \in \mathcal{L}\}$

- $\mathcal{R}_{2 a}=\left\{(x, y) \in \mathcal{P} \times \mathcal{P} \mid \mathrm{d}(x, y)=2\right.$ and $\left.\langle x, y\rangle \in \mathcal{T}_{a}\right\}$

- $\mathcal{R}_{2 b}=\left\{(x, y) \in \mathcal{P} \times \mathcal{P} \mid \mathrm{d}(x, y)=2\right.$ and $\left.\langle x, y\rangle \in \mathcal{T}_{b}\right\}$;

- $\mathcal{R}_{2 c}=\{(x, y) \in \mathcal{P} \times \mathcal{P} \mid \mathrm{d}(x, y)=2$ and $\langle x, y\rangle$ is a grid-quad $\}$;

- $\mathcal{R}_{3}=\{(x, y) \in \mathcal{P} \times \mathcal{P} \mid \mathrm{d}(x, y)=3\}$.

Suppose $t_{a} \neq t_{b}$. Then the eigenvalues of $A$ are $-(t+1)=-t_{a}-t_{b}-1$, $-t_{b}-1+s,-t_{a}-1+s, s-2,2 s-1, s t_{a}+s-1, s t_{b}+s-1$ and $s t_{a}+$ $s t_{b}+s$, with respective multiplicities $d_{\mathcal{S}}=\frac{s^{3}\left(s t_{a}+1\right)\left(s t_{b}+1\right)}{\left(s+t_{a}\right)\left(s+t_{b}\right)}, \frac{s^{2} t_{a}\left(s t_{a}+1\right)\left(s t_{b}+1\right)}{\left(s+t_{a}\right)\left(s+t_{b}\right)}$, $\frac{s^{2} t_{b}\left(s t_{a}+1\right)\left(s t_{b}+1\right)}{\left(s+t_{a}\right)\left(s+t_{b}\right)}, s^{2} t_{a} t_{b}, \frac{s t_{a} t_{b}\left(s t_{a}+1\right)\left(s t_{b}+1\right)}{\left(s+t_{a}\right)\left(s+t_{b}\right)}, s t_{b}, s t_{a}$ and 1. The algebra $\overline{\mathcal{A}}=$ $\mathcal{A}=\left\langle A_{0}, A_{1 a}, A_{1 b}, A_{1 c}, A_{2 a}, A_{2 b}, A_{2 c}, A_{3}\right\rangle$ is 8-dimensional and closed under Hadamard multiplication.

Suppose $t_{a}=t_{b}$. Then the eigenvalues of $\mathcal{S}$ are $-(t+1)=-2 t_{a}-1$, $-t_{a}-1+s, s-2,2 s-1, s t_{a}+s-1$ and $2 s t_{a}+s$, with respective multiplicities equal to $d_{\mathcal{S}}=\frac{s^{3}\left(s t_{a}+1\right)^{2}}{\left(s+t_{a}\right)^{2}}, \frac{2 t_{a} s^{2}\left(s t_{a}+1\right)^{2}}{\left(s+t_{a}\right)^{2}}, t_{a}^{2} s^{2}, \frac{s t_{a}^{2}\left(s t_{a}+1\right)^{2}}{\left(s+t_{a}\right)^{2}}, 2 s t_{a}$ and 1 . The algebra $\overline{\mathcal{A}}=\mathcal{A}=\left\langle A_{0}, A_{1 a}+A_{1 b}, A_{1 c}, A_{2 a}+A_{2 b}, A_{2 c}, A_{3}\right\rangle$ is 6-dimensional and closed under Hadamard multiplication.

\section{Proofs of Theorems 3 and 4}

In this section, we suppose that $\mathcal{S}=(\mathcal{P}, \mathcal{L}, \mathrm{I})$ is a finite near hexagon of order $(2, t)$. As there are points incident with at least two lines, we should have $t \geq 1$. 
Lemma 1 If $x$ and $y$ are two points of $\mathcal{S}$, then every shortest path between $x$ and $y$ can be extended to a shortest path between $x$ and a point $z$ at distance 3 from $x$.

Proof. This is obvious if $\mathrm{d}(x, y)=3$. If $\mathrm{d}(x, y)=1$, then every line through $y$ distinct from $x y$ contains a point of $\Gamma_{2}(x)$. So, it suffices to prove the lemma in the case $\mathrm{d}(x, y)=2$.

Suppose that $\mathrm{d}(x, y)=2$ and there are no points in $\Gamma_{1}(y) \cap \Gamma_{3}(x)$. Each of the $t+1 \geq 2$ lines through $y$ then contains a unique point of $\Gamma_{1}(x)$. By Proposition 2.5 of Shult and Yanushka [17, we then know that $x$ and $y$ are contained in a unique quad $Q$. As all lines through $y$ are contained in $Q$, this quad must have order $(2, t)$. But that is impossible. If we take a point $w \in \mathcal{P} \backslash Q$ that is collinear with a point $w^{\prime} \in Q$, then $w^{\prime}$ would be incident with at least $t+2$ lines $\left(t+1\right.$ in $Q$ and the extra line $\left.w^{\prime} w\right)$. So, if $\mathrm{d}(x, y)=2$, then there exists a $z \in \Gamma_{1}(y) \cap \Gamma_{3}(x)$.

A hyperplane of $\mathcal{S}$ is defined as a proper subset $X$ of $\mathcal{P}$ that intersects each line in a singleton or the whole line.

Lemma 2 If $x$ and $y$ are two distinct points of $\mathcal{S}$, then there exists a hyperplane containing $y$, but not $x$.

Proof. By Lemma 1, we can extend every shortest path between $x$ and $y$ to a shortest path between $x$ and a point $z$ at distance 3 from $x$. Let $H$ denote the set of points of $\mathcal{S}$ at distance at most 2 from $z$. Then $H$ is a hyperplane of $\mathcal{S}$ containing $y$, but not $x$.

Lemma 3 The near hexagon $\mathcal{S}$ has full projective embeddings.

Proof. As every line of $\mathcal{S}$ is incident with precisely three points, we know by Corollary 4 on page 184 of [16 that $\mathcal{S}$ has full projective embeddings if and only if for every two distinct points $x$ and $y$, there exists a hyperplane of $\mathcal{S}$ containing $y$, but not $x$. In view of Lemma 2, we thus see that $\mathcal{S}$ must have full projective embeddings.

Since $\mathcal{S}$ has full projective embeddings, we know from [16] that $\mathcal{S}$ should also have an absolutely universal embedding $\widetilde{\epsilon}: \mathcal{S} \rightarrow \mathrm{PG}(\widetilde{V})$. This absolutely universal embedding can be defined as follows. Let $V$ be a vector space having a basis $\left\{\bar{v}_{x} \mid x \in \mathcal{P}\right\}$ indexed by the points of $\mathcal{S}$. Let $W$ be the subspace of $V$ generated by all vectors $\bar{v}_{x}+\bar{v}_{y}+\bar{v}_{z}$, where $\{x, y, z\}$ is a line of $\mathcal{S}$. Consider then the quotient vector space $\widetilde{V}:=V / W$ and put $\widetilde{\epsilon}(x):=\left\langle\bar{v}_{x}+W\right\rangle \in \operatorname{PG}(V / W)$ for every point $x$ of $\mathcal{S}$.

This construction of $\widetilde{\epsilon}$ implies that the embedding rank $r$ of $\mathcal{S}$ is equal to $v-\operatorname{rank}_{\mathbb{F}_{2}}(M)$, where $v=|\mathcal{P}|$ and $M$ is the incidence matrix of $\mathcal{S}$. We have

$r=v-\operatorname{rank}_{\mathbb{F}_{2}}(M) \geq v-\operatorname{rank}_{\mathbb{R}}(M)=v-\operatorname{rank}_{\mathbb{R}}\left(M M^{T}\right)=v-\operatorname{rank}_{\mathbb{R}}(A+(t+1) I)=m$,

where $A$ is the collinearity matrix of $\mathcal{S}$ and $m$ is the multiplicity of the eigenvalue $-(t+1)$ of $A$. By Theorem 1 (3), we know that $m \geq d_{\mathcal{S}}$. Hence, $r \geq d_{\mathcal{S}}$. 
Moreover, if $r=d_{\mathcal{S}}$, then the multiplicity $m$ of the eigenvalue $-(t+1)$ of $A$ should be equal to $d_{\mathcal{S}}$. This finishes the proof of Theorem 3 The following proposition is precisely Theorem 4

Proposition 2 The near hexagon $\mathcal{S}$ has non-full projective embeddings in $\mathrm{PG}\left(d_{\mathcal{S}}-1, \mathbb{R}\right)$.

Proof. Consider the matrix $N$ as defined in Section 3 whose rows and columns are indexed by the points in accordance with the ordering $p_{1}, p_{2}, \ldots, p_{v}$ of these points. Since $N^{2}=\alpha N$ with $\alpha>0$, we see that the matrix $N$ is positive semi-definite. As the rank of $N$ is equal to $d_{\mathcal{S}}$, we then know that $N$ is the Gram matrix ([14, Section 7.2]) of a collection $\bar{w}_{1}, \bar{w}_{2}, \ldots, \bar{w}_{v}$ of vectors in a $d_{\mathcal{S}}$-dimensional Euclidean space $E$ such that $\left\langle\bar{w}_{1}, \bar{w}_{2}, \ldots, \bar{w}_{v}\right\rangle=E$. So, if $i, j \in\{1,2, \ldots, v\}$, then $N_{i j}=\bar{w}_{i} \cdot \bar{w}_{j}$. Since all diagonal entries of $N$ are equal to 1 , all $\bar{w}_{i}$ 's have norm 1 . As $\bar{w}_{i} \cdot \bar{w}_{j} \notin\{1,-1\}$ for $i \neq j$, we see that any two distinct vectors in the collection are linearly independent.

Suppose $\left\{p_{i}, p_{j}, p_{k}\right\}$ is a line of $\mathcal{S}$. From $\bar{w}_{i} \cdot \bar{w}_{i}=\bar{w}_{j} \cdot \bar{w}_{j}=\bar{w}_{k} \cdot \bar{w}_{k}=1$ and $\bar{w}_{i} \cdot \bar{w}_{j}=\bar{w}_{i} \cdot \bar{w}_{k}=\bar{w}_{j} \cdot \bar{w}_{k}=-\frac{1}{2}$, it follows that $\left(\bar{w}_{i}+\bar{w}_{j}+\bar{w}_{k}\right) \cdot\left(\bar{w}_{i}+\bar{w}_{j}+\bar{w}_{k}\right)=0$, i.e. $\bar{w}_{i}+\bar{w}_{j}+\bar{w}_{k}=\bar{o}$. This implies that the map $p_{i} \mapsto\left\langle\bar{w}_{i}\right\rangle$ defines a non-full embedding of $\mathcal{S}$ in $\operatorname{PG}(E) \cong \operatorname{PG}\left(d_{\mathcal{S}}-1, \mathbb{R}\right)$.

\section{References}

1. M. Aschbacher. Flag structures on Tits geometries. Geom. Dedicata 14 (1983), 21-32.

2. A. E. Brouwer, A. M. Cohen, J. I. Hall and H. A. Wilbrink. Near polygons and Fischer spaces. Geom. Dedicata 49 (1994), 349-368.

3. A. E. Brouwer, A. M. Cohen and A. Neumaier. Distance-regular graphs. Springer-Verlag, 1989.

4. A. E. Brouwer and H. A. Wilbrink. The structure of near polygons with quads. Geom. Dedicata 14 (1983), 145-176.

5. B. De Bruyn. On near hexagons and spreads of generalized quadrangles. J. Algebraic Combin. 11 (2000), 211-226.

6. B. De Bruyn. Near hexagons with four points on a line. Adv. Geom. 1 (2001), 211-228.

7. B. De Bruyn. Dense near octagons with four points on each line, III. Ann. Comb. 15 (2011), 19-35.

8. B. De Bruyn. On tetrahedrally closed line systems and a generalization of the HaemersRoos inequality. Submitted, 2015, http://cage.ugent.be/geometry/preprints.php.

9. B. De Bruyn. Computations regarding the paper "On the adjacency algebras of near hexagons with an order". Online available documents, http://cage.ugent.be/ geometry/preprints.php

10. B. De Bruyn and F. Vanhove. On $Q$-polynomial regular near $2 d$-gons. Combinatorica 35 (2015), 181-208.

11. S. Dixmier and F. Zara. Etude d'un quadrangle généralisé autour de deux de ses points non liés. Preprint 1976.

12. A. J. Hoffman. On the polynomial of a graph. Amer. Math. Monthly 70 (1963), 30-36.

13. R. A. Horn and C. R. Johnson. Topics in matrix analysis. Cambridge University Press, 1994.

14. R. A. Horn and C. R. Johnson. Matrix analysis. Cambridge University Press, 2013.

15. S. E. Payne and J. A. Thas. Finite generalized quadrangles. European Mathematical Society, 2009.

16. M. A. Ronan. Embeddings and hyperplanes of discrete geometries. European J. Combin. 8 (1987), 179-185.

17. E. E. Shult and A. Yanushka. Near $n$-gons and line systems. Geom. Dedicata 9 (1980), $1-72$ 